\title{
Medical Case Reports Journal
}

\section{Dermatomyositis and Breast Cancer: A Case Report and Review}

\author{
Garcia Fuentes Carolina Lisbeth ${ }^{1}$, Rodrigo Collado Chagoya ${ }^{2 *}$, Hernández Romero Javier ${ }^{2}$, Eliosa Alvarado \\ Gumaro Alejandro ${ }^{2}$, Velasco Medina Andrea ${ }^{2}$, Velázquez Sámano Guillermo ${ }^{2}$, Castelazo Rico German ${ }^{3}$ and \\ Isabel Flores Garcia ${ }^{1}$
}

${ }^{1}$ Department of Gynecology and Obstetrics, Instituto Mexicano Seguro Social Hospital General Zona, Mexico

${ }^{2}$ Department of Clinical Immunology and Allergy, Hospital General De México, Mexico

${ }^{3}$ Department of Oncological Gynecology, Instituto Mexicano Seguro Social “Centro Medico Nacional La Raza”, Mexico

Article info

Received 08 February 2018

Revised 12 February 2018

Published 16 February 2018

${ }^{*}$ Corresponding author: Rodrigo

Collado Chagoya, Department of

Clinical Immunology and Allergy,

Hospital General de México,

Mexico;

E-mail: rodnova87@hotmail.com

\begin{abstract}
Breast cancer is the leading cause of death from malignancy in women worldwide, with nearly 500,000 deaths each year. Its diagnosis must be a priority for world health. Breast cancer is the second tumor that can produce a paraneoplastic syndrome after lung cancer. Dermatomyositis with a diagnosis in later ages correlates significantly with an underlying malignancy. Therefore, you should always rule out the presence of a common disease (breast cancer) with the presentation of a rare disease (dermatomyositis).
\end{abstract}

Keywords: Dermatomyositis; Breast cancer; Paraneoplastic syndrome.

\section{Introduction}

Dermatomyositis is an idiopathic inflammatory disease that mainly affects the skeletal muscle and the skin with characteristic skin lesions (heliotrope, Gottron papules). The estimated incidence of dermatomyositis is approximately $1 / 100,000$, can affect children and adults, and is much more common in women than in men $(2: 1)$ [1,2]. Initial presentation follows a bimodal distribution between ages 5-15 and 45-64 and tends to progress over a 3 to 6-month period before the patient will seek medical attention. Most cases are idiopathic, dermatomyositis (DM) is associated with an underlying malignancy in 6-60\% of cases, and in which case, it is considered a paraneoplastic syndrome [3]. The types of cancer most associated with dermatomyositis in adult women are breast cancer and ovarian cancer, reporting a frequency of $20-36 \%$ in adults with breast cancer in dermatomyositis [4]. In the mini review, we try to shortly review the recent developments and applications of carbon-based magnetic and fluorescent nanohybrids as multi-modal imaging agents.
The pathogenic relationship between dermatomyositis and cancer is not completely explained. Apparently, the regeneration cells that appear in muscles with myositis express high levels of myositis-specific antigens, and it is these same antigens that are expressed in several types of cancer associated with inflammatory myopathies. The union between cancer and dermatomyositis seems to be the expression of antigens common to cancer and muscle tissues in some patients with dermatomyositis [5].

DM typically presents with progressive, symmetrical, proximal muscle weakness and characteristic skin lesions such as helitrope rash, Gottron's papules, Gottron's sign, the V-sign and shawl sign. Additional cutaneous manifestations that have become more commonly recognized include vasculopathic changes (i.e. telangiectasias and livedo reticularis), cuticular overgrowth ('mechanic's hands') and poikiloderma [6]. In the cases of typical DM associated with breast cancer, it has been established a parallel course with breast 
cancer evolution after surgery, local radiotherapy and systemic therapy with cytotoxic agents or hormones [7].

Contrary to this typical presentation, the presence of rapid progression of symptoms over a much shorter period and skin manifestations without development of muscular deterioration can suggest an underlying malignancy. These atypical presentation so-called amyopathic DM, is associated with breast cancer, characterized by the absence of muscular symptoms. It has been suggested that amyopathic DM is not responsive either to steroid treatment or to specific treatment of the underlying malignancy $[8,9]$.

It has also been observed that myopathy may relapse in the setting of recurrent malignancy, further supporting a paraneoplastic origin of malignancy-associated DM [10]. Paraneoplastic dermatomyositis can precede, coincide or develop after the diagnosis of cancer. The diagnosis of paraneoplastic dermatomyositis should be made under medical suspicion and especially in patients with delayed presentation dermatomyositis [11,12].

The risk factors of malignancy in the series of patients with dermatomyositis and/or polymyositis had been studied by many clinicians from various countries. An extensive meta-analysis of clinical trials found older age, male sex, dysphagia, cutaneous necrosis, cutaneous vasculitis, rapid onset ( $<4$ weeks), elevated CK, higher ESR, and higher CRP as factors to be associated with higher risk [13]. Currently, the association between anti-155/140 antibodies and paraneoplastic dermatomyositis has been reported, finding a sensitivity of $50 \%$ and a specificity of $96 \%$ for the detection of cancer associated with dermatomyositis. It has been similarly correlated that the presence of "routine antibodies" (antibodies against Jo-1, Anti Ro-52, U1RNP, U3-RNP, Ku and PM SCL) increase the relative cancer risk of 6-7 in contrariety with patients with negative antibodies $[14,15]$.

We present this case as a reminder that a common disease (breast cancer) may have an uncommon presentation (Dermatomyositis as paraneoplastic syndrome), which is why an underlying neoplastic process should always be considered in patients with delayed presentation dermatomyositis.

\section{Case Report}

A 53-year-old woman with 6 weeks with progressive proximal muscle weakness (scapular and pelvic belt) of symmetric distribution, as well as skin lesions categorized violaceous rash of the upper back, chest, the orbital region and in the neck region (Figures 1 and 2).

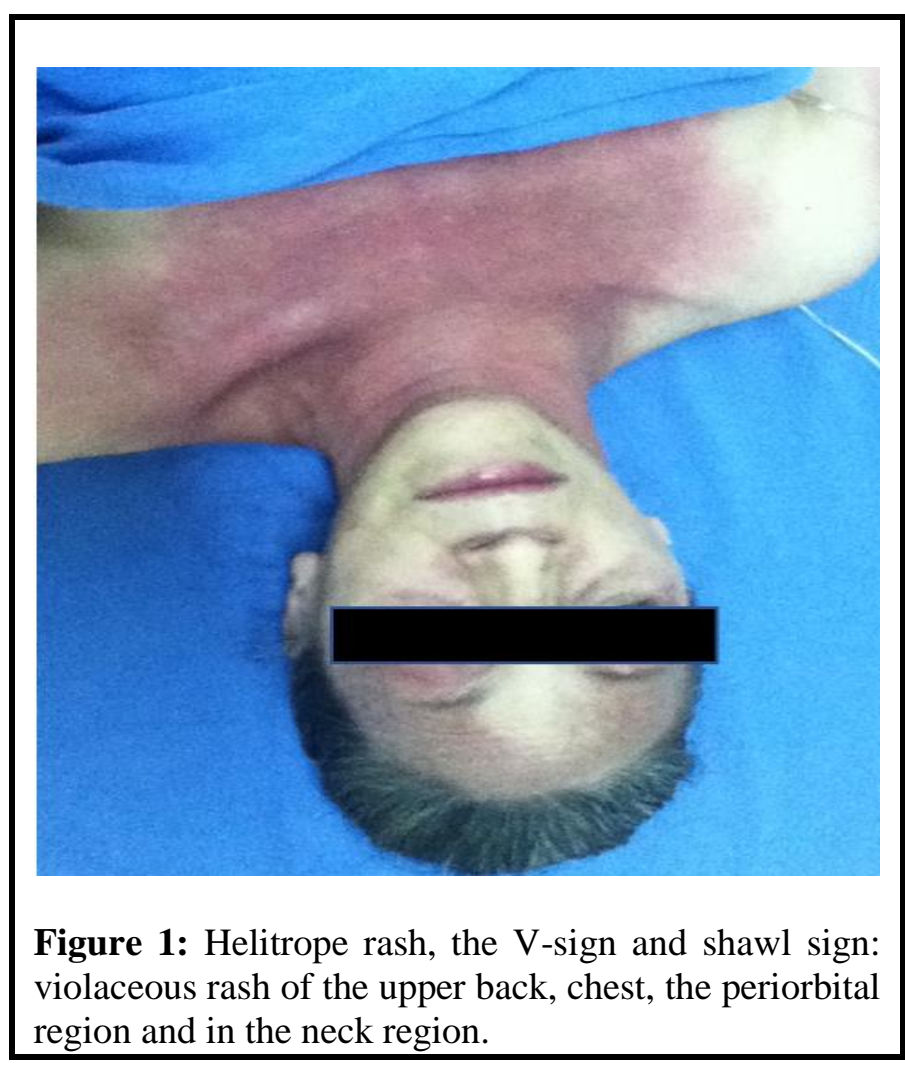

Within 8 weeks also referred weight loss of 10 kilograms. Within 1 week began with dysphagia and increased muscle weakness.

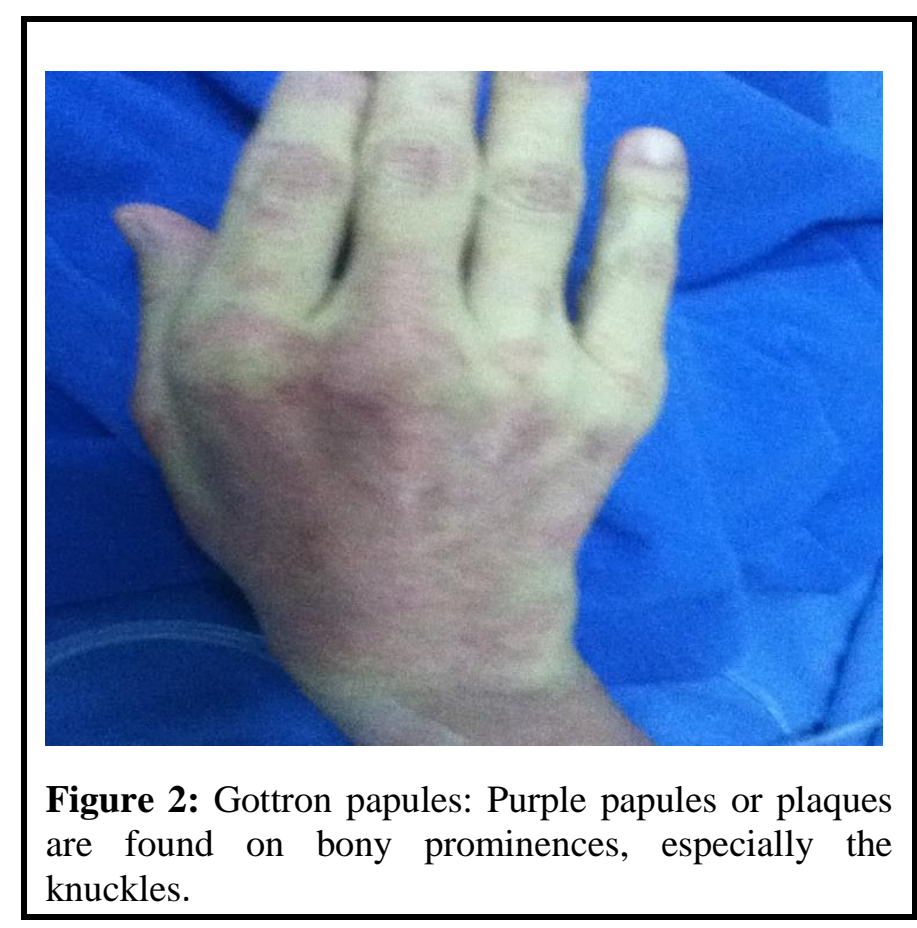

Her dermatology exam showed heliotrope rash, Gottron papules, periungual erythema, abnormal nail-fold capillaries, and diffuse violaceous rash throughout her body. On breast exam, she had a mass in the right breast of approximately $1.5 \times 2 \mathrm{~cm}$ not mobile, stick to deep 
planes located in the upper external quadrant and presence of positive axillary nodes in the right axilla, with a size of 0.2 to $0.5 \mathrm{~cm}$.

Based on the data of proximal myopathy and the skin lesions found, a diagnosis protocol of inflammatory myopathy was initiated, presenting an alteration in muscle enzymes with TGO (78 U/l), TGP (73 U/l), CPK (450 U/l), LDH (860 U/l), myopathic pattern in electromyography study, histopathological study of skin biopsy (perivascular and perimysial inflammatory infiltrate with predominance of lymphocytes) (Figure 3). The immunological profile showed (ANA 1:640 fine mottled pattern, Anti Ro-52 Positive ${ }^{++}$, Anti Jo-1 Neg., Anti Ku-2 Neg., Anti SSP Neg., Anti Mi-2 Neg.,) performing the diagnosis of dermatomyositis and was prescribed prednisone $50 \mathrm{mg}$ daily.

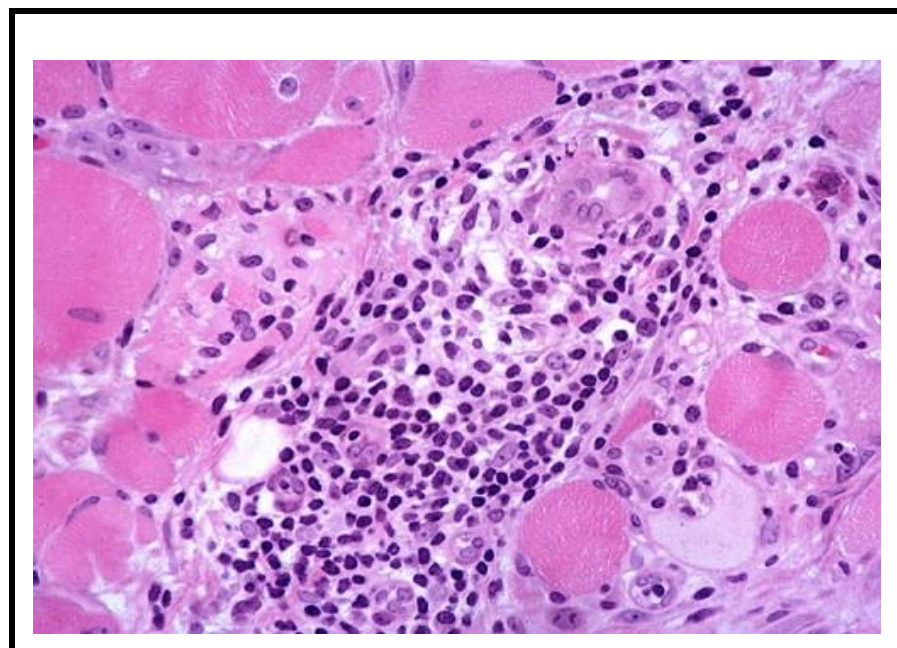

Figure 3: Inflammation, vasculitis and peri-fascicular atrophy with lymphocytic infiltrate.

Based on gynecological findings, a mammogram was performed, finding calcifications suggestive of malignancy in the right breast and an area of focal asymmetry in the upper outer quadrant (Figure 4). A right breast biopsy showed a multifocal invasive ductal carcinoma with axillary affectation, counting with negative estrogen receptors, negative progestin receptors, negative Her2/neu. Making the diagnosis of breast cancer and staging as T1N1MO. Based on the stage of the tumor, a modified radical mastectomy was performed and sent to medical oncology for the initiation of chemotherapy with docetaxel.

In the control review 3 months after the surgical procedure, her skin rash and muscle weakness have improved, but are not yet back to normal. She was started on oral weekly methotrexate as a steroid-sparing agent and continues in surveillance by the service of immunology, oncological gynecology, rheumatology and dermatology.

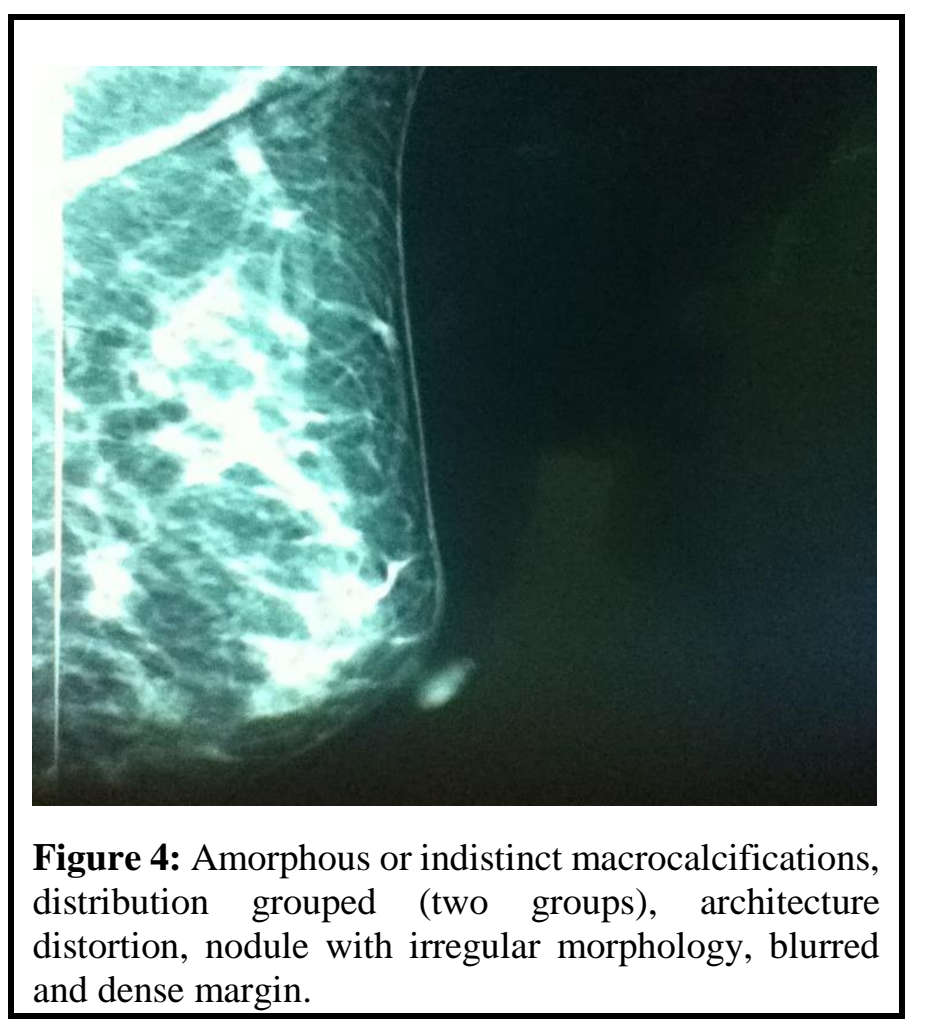

\section{Discussion}

Dermatomyositis is an idiopathic inflammatory muscle disease of autoimmune component characterized by the presence of cutaneous lesions such as heliotrope rash and Gottron nodules accompanied by the presence of proximal muscular weakness, being able to find other symptoms such as dysphagia, regurgitation or bronchial aspiration pneumonia [16].

The association of Dermatomyositis (DM) or polymyositis (PM) with malignant tumors was suggested since 1916. Dermatomyositis is associated with an underlying malignant disease in 6-60\% of cases, being considered in this case a paraneoplastic syndrome. The risk of malignancy is higher in patients aged between 45 and 74 years old at the age of diagnosis [16].

The presence of positive antibodies has been more associated with the presence of underlying malignancy. The AntiRo-52 antibody is the most frequent of the antibodies associated with myositis (>30\%), being frequently accompanied by specific antibodies of myositis (Anti Jo-1) or other antibodies (ANA). It has been reported that similarly it has been associated with an increased risk of cancer and interstitial lung disease. [17]. The relationship between DM and cancer has been described in different retrospective studies. It is 
estimated that the incidence of cancer in patients with DM is between $15-30 \%$ of the total of cases. The tumors most commonly associated are breast, ovarian, gastrointestinal, lung and lymphoma, varying in frequency in the series reviewed [17].

Paraneoplastic dermatomyositis can precede, coincide or develop after the diagnosis of cancer. Most patients were diagnosed with cancer before they were diagnosed with DM and normally the presence of DM was associated with late-stage tumors; but there are cases in which the presentation of dermatomyositis may precede the onset of breast cancer the so the diagnosis should be based on a previous suspicion of the disease and subsequent surveillance $[5,18]$. The most common type of breast cancer associated with dermatomyositis was invasive ductal carcinoma. The distribution of triplenegative, her2-positive, and estrogen or progesterone receptor-positive breast cancers was relatively even [18].

The ultimate treatment of paraneoplastic dermatomyositis is to remove the primary neoplasm. This will result in the resolution of dermatomyositis in most cases, and in a minority of cases additional immunomodulatory therapies are required [19]. Malignancy associated dermatomyositis has a poor prognosis. Cancer significantly decreases survival, as one study found patients with DM and malignancy had a 5-year survival of only $10 \%$ [19].

\section{Conclusion}

Breast cancer is the leading cause of death from malignancy in women worldwide, (nearly 500,000 deaths each year). Its diagnosis must be a priority for world health. Breast cancer is the second tumor that can produce a paraneoplastic syndrome after lung cancer. Dermatomyositis with a diagnosis in later ages correlates significantly with an underlying malignancy. Therefore, you should always rule out the presence of a common disease (breast cancer) with the presentation of a rare disease (dermatomyositis).

\section{Conflict of Interest}

None declared.

\section{Funding}

None declared.

\section{References}

1. Bohan A, Peter JB. Polymyositis and dermatomyositis (first of two parts). N Engl J Med 1975; 292: 344-347.
2. Santmyire-Rosenberger B, Dugan EM. Skin involvement in dermatomyositis. Curr Opin Rheumatol 2003; 15: 714-722.

3. Zhang W, Jiang S, Huang L, et al. Dermatomyositis and malignancy: A retrospective study of 115 cases. Eur Rev Med Pharmacol Sci 2009; 13: 77-80.

4. Levine D, Miller S, Al-Dawsari $\mathrm{N}$, et al. Paraneoplastic dermatoses associated with gynecologic and breast malignancies. Obstet Gynecol Surv 2010; 65: 455-461.

5. Yeh CN, Chen SC, Hwang TL, et al. Breast carcinoma in patients with dermatomyositis: A retrospective analysis of eight cases. Chang Gung Med J 2002; 25: 374-380.

6. Levine SM. Cancer and myositis: New insights into an old association. Curr Opin Rheumatol 2006; 18: 620-624.

7. Goyal S, Nousari HC. Paraneoplastic amyopathic dermatomyositis associated with breast cancer recurrence. J Am Acad Dermatol 1999; 41: 874875.

8. Osako T, Ito Y, Morimatsu A. Flare-up of dermatomyositis along with recurrence of breast cancer. Breast J 2007; 13: 200-202.

9. Dias LP, Faria AL, Scandiuzzi MM, et al. A rare case of severe myositis as paraneoplastic syndrome on breast cancer. World J Surg Oncol 2015; 13: 134.

10. Ahuja S, Makkar P, Gupta S, et al. Paraneoplastic syndrome and underlying breast cancer: A worsening rash despite initiation of chemotherapy. J Community Support Oncol 2016; 14: 229-231.

11. Danko K, Ponyi A, Constantin T, et al. Long-term survival of patients with idiopathic inflammatory myopathies according to clinical features: A longitudinal study of 162 cases. Medicine (Baltimore) 2004; 83: 35-42.

12. Andras C, Ponyi A, Constantin T. Dermatomyositis and polymyositis associated with malignancy: A 21-year retrospective study. J Rheumato 2008; 35: 438-444.

13. Wakata N, Kurihara T, Saito E, et al. Polymyositis and dermatomyositis associated with malignancy: A 30-year retrospective study. Int J Dermatol 2002; 41: 729-734.

14. Kaji K, Fujjimoto M, Hasegawa M. Identification of a novel autoantibody reactive with 155 and $140 \mathrm{kDa}$ nuclear proteins in patients with 
dermatomyositis: An association with malignancy. Rheumatol 2007; 46: 25-28.

15. Chinoy H, Fertig N, Oddis CV. The diagnostic utility of myositis autoantibody testing for predicting the risk of cancer-associated myositis. Ann Rheum Dis 2007; 66: 1345-1349.

16. Chen YJ, Wu CY, Huang YL. Cancer risks of dermatomyositis and polymyositis: A nationwide cohort study in Taiwan. Arthritis Res Ther 2010; 12: R70.

17. Yang H, Peng Q, Yin L. Identification of multiple cancer-associated myositis-specific autoantibodies in idiopathic inflammatory myopathies: a large longitudinal cohort study. Arthritis Res Ther 2017; 19: 259.

18. Sandhu NP, Zakaria S, Degnim AC, et al. Dermatomyositis presenting as a paraneoplastic syndrome due to underlying breast cancer. BMJ Case Rep 2011.

19. Papakonstantinou E, Raap U. Sudden onset of dermatomyositis as a sign of recurrence of breast cancer along with regional metastasis. J Autoimmun Res 2016; 3: 1009.

This manuscript was peer-reviewed

Mode of Review: Single-blinded

Academic Editor: Dr. Ibrahim Anwar Ahmed Abdelazim

Copyright: (02018 Garcia FCL, et al. This article is distributed under the terms of the Creative Commons Attribution 4.0 International License (http://creativecommons.org/licenses/by/4.0/), which permits unrestricted use, distribution, and reproduction in any medium, provided you give appropriate credit to the original author(s) and the source, provide a link to the Creative Commons license, and indicate if changes were made. 